\title{
CpG Oligodeoxynucleotide Developed For Activating Primate Immune Responses Promotes Anti-Tumoral Effect Combine With Neo-Antigen Based mRNA Cancer Vaccine
}

\section{Rongkuan Hu ( $\nabla$ rkhu@mail.ustc.edu.cn )}

University of Science and Technology of China https://orcid.org/0000-0001-6293-2004

\section{Qin Li}

GenePharma Co., Ltd, Suzhou, 215125, China

Jie Ren

Department of General Surgery, The Second Affiliated Hospital, Soochow University, Suzhou 215004, Jiangsu, China

Wei Liu

Department of Pathology, the First Affiliated Hospital of Soochow University. Suzhou, 215006, China Guoqin Jiang

Department of General Surgery, The Second Affiliated Hospital, Soochow University, Suzhou 215004, Jiangsu, China

\section{Research}

Keywords: $\mathrm{CpG}$, IFN- $\gamma$, TLR9, mRNA vaccine, neo-antigen

Posted Date: January 20th, 2021

DOI: https://doi.org/10.21203/rs.3.rs-148613/v1

License: (c) (i) This work is licensed under a Creative Commons Attribution 4.0 International License. Read Full License 


\section{Abstract}

Synthetic phosphorthiolate modified CPG-oligodeoxynucleotides (CPG-ODN) activate innate and adaptive immune responses, which being exploited as a therapeutic approach. Here, we first screened and identified a new CpG-B class ODN (CpG2018B) that effectively stimulates type II interferon both in mouse Plasmacytoid dendritic cells ( $p-D C$ ) and human PBMC. In addition, CpG2018B promotes cytokine production mainly via toll-like receptor 9 (TLR9) pathways. We further demonstrated that intratumoral (IT) injection of CpG2018B inhibits melanoma growth in syngeneic models and could turn "cold" tumors into "hot" tumors. Then, CpG2018B and mRNA based neo-antigen cancer vaccine were encapsulated into lipid-nanoparticle (LNP) and intratumoral injected into melanoma mice models. Interestingly, vaccination with $\mathrm{CpG}$ or mRNA vaccine alone could inhibit tumor growth respectively, while, $\mathrm{CpG}$ combine with mRNA vaccine enhanced the anti-tumor effect. At last, we described the long-term safety and tolerability of CpG2018B and mRNA therapy in mice models. In conclusion, we identified a novel CpG-B ODNs to promote immune response and $\mathrm{CpG}$ combine with mRNA cancer vaccines are attractive candidate for immune stimulatory sequences (ISS) based therapeutic strategies.

\section{Introduction}

Toll-like receptors (TLRs) constitute a set of receptors that recognize pathogen associated molecular from microorganisms and danger related molecules of a host, and induce protective immune responses against these infections[1, 2]. More than ten TLRs have been identified both in mammalian and nonmammalian species. Briefly, TLR3 is activated by double stranded RNA (dsRNAs)[3], while TLR7 and TLR8 are often activated by nucleosides and viral single stranded RNAs (ssRNAs)[4]. TLR9 recognized the unmethylated $\mathrm{CpG}$ present in bacteria or synthetic ODNs with unmethylated $\mathrm{CpG}$ motif[5]. The other TLRs are cell-surface TLRs. They are located on the cell or plasma membrane and recognize cell constituents such as lipoproteins, flagellin and lipopeptides.

Among these TLRs, TLR9 can induce Th1 type immune response by bacterial and synthetic DNA containing unmethylated $\mathrm{CpG}$ motifs[6]. The immune activities are multifactorial and depend on the ODNs backbone, sequence and the number of CpG motifs. CPG oligodeoxynucleotides (ODNs) also known as immunostimulatory sequences (ISS) have received widespread attention recently after approved by FDA as vaccine adjuvants and reported as strong candidates for therapeutic immunepotentiates[7]. CPG ODNs are composed of phosphorthiolate modified residues and require at least one or two CpG nucleotides. Based on the sequence, structure and immune profiles induced, CPG ODNs have been described into several classes including class A, B and C. Class B strongly activates B cell function and induces plasmacytoid dendritic cells (pDCs) to release IFN- $y$ and TNF- $a$. Class A containing a CpG within a self-complementary (palindrome) strongly induces IFN-a secretion but poorly promotes $\mathrm{B}$ cell proliferation. CpG-C requires at least one CG motif and contains at least two or more CpG dinucleotides. CpGs are excellent candidates for ISS-based therapies[8, 9]. 
In the last decades, more than 100 clinical trials are mainly focused on immune-oncology or adjuvants of these types CpG ODNs, including CpG7909 (Pfizer), SD101 (Dynavax), MGN1703 (Mologen), CMP-001 (Checkmate) and IMO-2125 (Idera). In 2017, a CpG ODN in combination with vaccines designed to prevent hepatitis B (HBV) was approved by the US FDA, which was the first commercialized CpG treatment (HEPLISAV)[7]. Moreover, several CpG combination therapies are still in phase II/III, including CpG plus rituximab, CpG combines with PD-1 or with OX40 antibody (NCT02521870, NCT03410901, etc.). Wang et al. showed that intratumoral injection of CpG ODN reverting resistance to PD-1 blockade treatment[10]; Combination of SD101 with OX40 antibody can trigger a T cell immune response, which inturn remarkably cure multiple types of cancer and prevent spontaneous genetically driven cancers[11].

mRNA therapeutics represent a promising alternative to conventional vaccine approaches because of their rapid development and low-cost manufacture and easy administration. The first study of the use of in-vitro transcribed (IVT) mRNA in-vivo was reported in 1990's[12, 13]. However, this promising result did not lead to substantial investment in mRNA therapeutics owing to mRNA instability, innate immunogenicity and inefficient delivery. Very recently, the use of chemical modified mRNA including pseudouridine $(\mathrm{pU}), 2$-thiouridine $(\mathrm{s} 2 \mathrm{U})$ and 5-methyl-cytidine $(\mathrm{m} 5 \mathrm{C})$ has beneficial features to safety and stability concerns, leading several mRNA based clinical trial (NCT03313778, NCT03323398, etc.). In addition, lipid nanoparticles (LNPs) have been identified as an efficient delivery vehicle for systemic administration of mRNA. Unlike DNA plasmid and viral gene delivery based vaccines, mRNA dose not transit to the nucleus, not integrate into chromosomes, therefore lead insertional mutagenesis and oncogenesis. mRNA vaccines provide transient, half - life dependent protein expression, and can restore "undruggable" targets such as transcriptional factors and non-membrane proteins. Besides, mRNA based neo-antigen to mobilize immunity against cancer mutations has been reported[14, 15]. Intra-nodal vaccination with personalized $m R N A$ vaccine showed antitumor immunity. Therefore, the mRNA vaccine field is developing extremely rapidly and sets of preclinical \& clinical data have been accumulated over the past several years.

Here, we identified a new class B CpG (2018B), which stimulates IFN-y secretion and gene expression in both mouse pDCs and human PBMC. Then, anti-tumor effect of 2018B in melanoma mouse model was observed, which in-turn converts "cold tumors" into "hot tumors" by stimulating CD4 and CD8 positive T cells. Given that, Combined CpG with mRNA based neo-antigen encapsulated in lipid nanoparticle (LNP) were injected to prevent tumor growth. At last, we described the long-term safety and tolerability of $\mathrm{CpG}$ and mRNA therapy in mice models. These results indicate a new therapeutic approach using $\mathrm{CpG}$ and mRNA vaccine for cancer treatment.

\section{Materials And Methods}

\section{Materials}

RPMI-1640, Fetal Bovine Serum (FBS), and Collagen I was purchased from Sigma-Aldrich (St.Louis, USA). CpGs and mRNA vaccines were synthesized by GenePharma (Suzhou, China) (Table 1). All ELISA kits 
including IFN- - , IL-6, TNF-a were purchased from Abcam (MA, USA).

\section{Primary cell isolation and cell culture}

pDCs were isolated from 6-8 week old, female C57BL/6J mice (Vital River Laboratory Animal Technology Co., Ltd, Beijing, China) or C57BL/6-TLR9-/- (Shanghai Model organisms, Shanghai, China) using EasySepTM Mouse Plasmacytoid DC isolation kit (STEMCELL technologies, MA, USA). Human peripheral blood mononuclear cells (PBMCs) were isolated using SepMate kit following the manufacture's protocol (STEMCELL technologies, MA, USA). PBMCs were cultured in RPMI-1640 (Sigma) with 10\% fetal bovine serum (FBS, Sigma) at $37^{\circ} \mathrm{C}$ in $5 \% \mathrm{CO}_{2}$. B16-F10 cell lines were obtained from ATCC. Tumor cells were cultured in Dulbecco's modified Eagle's medium (DMEM) containing $10 \% \mathrm{FBS}$ at $37^{\circ} \mathrm{C}$ in $5 \% \mathrm{CO}_{2}$.

\section{Clinical blood samples}

Five healthy volunteers without diagnosed metastasis tumors were collected in 2018 (median age is 43). $10 \mathrm{ml}$ blood sample was collected from each person at the Second Affiliated Hospital of Soochow University. All of the blood samples were obtained with informed consent from each person and approval from the Ethics Committee of the Second Affiliated Hospital of Soochow University.

\section{IFN-y and cytokines production assay in-vitro}

pDCs or PBMCs ( $10^{6}$ cells) were isolated from mouse spleen and human whole blood using StemCell isolation kit separately. Cell stimulations were performed in 96-well plates, pDCs or PBMC were stimulated for 24 to 48 hours with control ODN or CpGs prior to supernatant harvest. Supernatant were centrifuged and filtered for IFN- $\gamma$, TNF- $\alpha$ and IL- 6 measurement using ELISA kit following the manufacture's protocol (Abcam, MA, USA).

\section{Western blotting and immunohistochemistry}

Mouse pDCs cells were stimulated with CpGs for 48 hours and lysed using SDS buffer (1\% SDS). Total proteins were measured with the BCA assay kit (Thermo Fisher Scientific). Equal amounts of protein samples were subjected to $10 \%$ SDS-PAGE and transferred to polyvinylidene difluoride membranes (Millipore, USA). The membranes were blotted with primary antibodies against p-ERK, P-P38, p-JNK and Actin at $4^{\circ} \mathrm{C}$ overnight $(1: 1000$, respectively). After incubation with the secondary antibody (1:5000), the proteins were developed and detected as previously described[16].

For immunohistochemistry (IHC) was performed according to our previous study[16]. Briefly, paraffin embedded samples were deparaffinized and incubated with rabbit CD4, CD8 or TLR9 antibodies (1:200) for $1 \mathrm{~h}$ at $37^{\circ} \mathrm{C}$. After washing with PBS, slides were incubated with goat-anti-rabbit secondary antibody (1:2500) and treated with 3-diaminobenzidine solution for 20 minutes.

\section{Quantitative real time PCR (qRT-PCR)}


Total RNA from cells or tissues was isolated using TRIzol (Thermal Fisher). One micrograms of RNA was reversing transcribed using SuperScript Reverse Transcriptase Kit (Thermal Fisher). qRT-PCR was performed using the power SYBR Green Master Kit (Applied Biosystems). The following primers were used as described in table 2 . The relative expression of each gene was calculated with delta-delta CT and normalized to the reference gene $\beta$-actin.

\section{mRNA production and LNP formulation}

Neo-antigen sequence for B16-F10 melanoma model in C57BL/ 6 was identified and reported previously[14]. M30 peptide sequence (DWENVSPELNSTDQP) was optimized into mRNA sequence (atgGACTGGGAGAATGTAAGTCCAGAACTTAATTCTACAGATCAACCCtaa) and then synthesized by in-vitro transcription(IVT) using T7 polymerase where the UTP was substituted with 1-methylpseudo UTP. The DNA template was incorporated 5' UTR and 3'UTR includes a poly-A tail as reported[17]. mRNA was further caped with cap 0 and cap 1 by using Vaccinia Capping Kit (NEB). LNP formulations were prepared using ionizable lipid (DLIN-MC3), DSPC, cholesterol and PEG at molar ratios of 50:10:38.5:1.5. The lipid mixture was combined with mRNA at a volume ratio of 3:1 using a microfluidic mixer (Precision Nanosystems, Vancouver, BC) as previously described[17]. The final product was filtered through a $0.22 \mu \mathrm{m}$ filter and stored in pre-sterilized vials frozen until use.

\section{Animal studies}

B16-F10 tumor cells $\left(5 \times 10^{5}\right)$ were injected subcutaneously at sites of the abdomen of C57BL/6J mice (6-8 weeks, female, Vital River Laboratory Animal Technology Co., Ltd, Beijing, China). When tumor size reached $50-100 \mathrm{~mm}^{3}$, mice were injected with $\mathrm{CpG}(2.5 \mathrm{mg} / \mathrm{kg})$ and/or mRNA vaccine encapsulated in LNP $(0.5 \mathrm{mg} / \mathrm{kg})$ twice a week. Tumor size was monitored every 2-3 days and calculated with volume (length $\mathrm{x}$ width $\left.{ }^{2}\right) / 2$. Mice were sacrificed when tumor size reached $3000 \mathrm{~mm}^{3}$. Tumors were photographed and stained with hematoxylin \& eosin (H\&E). All animal studies were approved by the animal ethics committee of GenePharma Co., Ltd (Suzhou, China).

\section{Clinical chemistry panel}

All C57BL/6J mice were sacrificed and blood samples were collected into a serum separator tubes and shipment to CAM-SU Genomic Resource Center of Soochow University for sample analysis. Clinical chemistry parameters were measured in a blinded manner by a standard chemistry analyzer (HITACHI 7100) as described by CAM-SU Genomic Resource Center.

\section{Statistical analysis}

All results were shown as the mean with SD. Significance test was performed using GraphPad Prism 6.0 (GraphPad Software Inc., CA, USA). Statistical significance of different groups was calculated with ANOVA or Student's t-test. A $p$ value $<0.05$ was considered significant. 


\section{Results}

\section{Identification of CpG2018B promotes IFN-y secretion both in mouse pDCs and human PBMC}

Several studies showed that synthetic CpG ODN with CG motif has a major impact on immune activation, but it has been unclear whether different $\mathrm{CpG}$ sequence and chemical modifications might display different immune effects. To evaluate the possibility, we tested a panel of CpG OND with phosphorthiolate backbone modifications and detected the IFN-r secretion both in mouse pDCs and human PBMCs (Table 1). Immunostimulatory activity of ODN without CG motif 1040ODN was set as negative control, CpG-1018 which was approved by FDA(Dynavax) and two other clinical used CpGs, like CpG 7909 and SD-101 were set as positive control. Among these ODNs, CpG2018A and CpG2018B showed a generally similar pattern of immune-stimulation activity in mouse pDCs. Of note, CpG2018B, which was unique in this panel for containing two CG-rich regions, showed preferential induction of IFN- $y$ synthesis (Fig 1A). Furthermore, 5 human PBMCs were isolated from 5 healthy volunteers by centrifugation through a Ficoll density gradient and incubated with $\mathrm{CpGs}$ for 48 hours. Consistent of the discovery, CpG2018B exhibits marked immunomodulatory activity in human PBMCs(Fig 1B-1F).

\section{CpG2018B stimulates IFN-Y and cytokines secretion via TLR9}

TLR9 are known to signal the activation of variety of cells of innate and adaptive immune systems. To determine the molecular mechanism of CpG2018B, we first detected the IFN-y expression in mouse pDCs incubated with different concentration of $\mathrm{CpGs}$. As a result, IFN-y secretion and mRNA expression stimulated by CpG2018B was presented a dose-dependent manner(Fig. 2A, 2B). In addition, CpG2018B also promotes two other cytokines expression including TNF-a and IL-6 (Fig. 2C). Furthermore, the phosphorylation of p38(Thr180/Tyr182), p- ERK(Thr202/Tyr204) and p-JNK (Thr183/Tyr185) were also accumulated(Fig. 2D). These results indicated CpG2018B promotes cytokines expression and activates MAPK signaling.

To further exploit the potential mechanism of CpG2018B, pDCs from TLR9 knockout mice (Shanghai Model organisms, Shanghai, China) were treated with $\mathrm{CpG} 2018 \mathrm{~B}(1 \mu \mathrm{g} / \mathrm{ml})$. As a result in figure $2 \mathrm{E}$ and $2 \mathrm{~F}$, IFN- $\gamma$ stimulated by CpG2018B in pDCs was abrogated by TLR9 knockout. In consistent, TNF-a and IL-6 level were also decreased in TLR9 knockout pDCs (Fig. 2G, 2H). These data suggest that CpG2018B promotes IFN- $y$, TNF-a and IL-6 secretion mainly via TLR9.

\section{In-suit vaccination with CpG2018B induces cytokine expression and tumor regression in melanoma mice models}

Considering CpGs stimulates IFN- $y$ secretion and may inhibit tumor proliferation in-vivo, we then explored whether CpG-2018B has the same effect. To begin with, we established syngeneic mouse models(B16F10) of melanoma and then injected CpG2018B into the tumor nodule. After intratumoral injection of CpGs (2.5mg/kg, twice a week), the tumor growth was significantly suppressed by CpG2018B(Fig 3A, 3B 
and 3C). Further, cytokines expression including IFN- $\gamma$, TNF- $\alpha$ and IL- 6 were detected by qPCR. As a result, CpG2018B significantly stimulates these genes expression in tumor samples (Fig 3D, 3E and 3F).

We then analyzed the intra-tumoral T cells for their expression of functional markers, we observed that CpG2018B could activate CD4 and CD8 positive T cells expression in the tumor microenvironment (TME) with accumulated TLR9 levels (Fig 3G). In addition, H\&E staining indicated there are few tumor cells in CpG treatment groups (Fig 3G). These results suggest that CpG ODN may turn "cold" tumors into "hot" tumors.

\section{CpG2018B combine with lipid nanoparticle (LNP) formulated mRNA vaccine enables efficient immunotherapy against melanoma}

In-vitro transcribed (IVT) mRNA has recently come into focus as a potential new type of drug to alter genetic information. These synthetic mRNAs can be engineered to express proteins by structurally resembling natural mRNA. Vaccination with mRNA is a promising strategy for cancer prevention and have been entered into clinical development $[12,18]$. In particular, mRNA vaccine targeting tumor-specific mutations (neo-antigen) are ideal targets for cancer immunotherapy. As reported previously, Sebastian K, etal mapped non-synonymous mutations of the B16F10 tumor by next-generation sequencing and vaccinated with such mRNA vaccines induces tumor control in mice[14]. There are growing evidences to suggest that adjuvant effects associated with stimulation of type I and type II interferon may support adaptive immune responses[19, 20]. Considering these facts, we hypothesized that CpG2018B could augment mRNA vaccine treatment and help to induce robust antitumor immune responses. To test this hypothesis, we implanted C57B/6J mice with B16-F10 cells which representing melanoma. mRNA targeting tumor specific antigens (M30) were incorporated into lipid nanoparticles (LNP) as previously described in methods. Then, $\mathrm{CpG}$ and $\mathrm{LNP}$ formulated $\mathrm{mRNA}$ vaccines were injected into syngeneic xenograft models twice a week. The mice were monitored for tumor growth and sacrificed when tumor size reached $3000 \mathrm{~mm}^{3}$. In figure $4 \mathrm{~A}$ to $4 \mathrm{C}$, tumors of $10400 \mathrm{DN}$ treated mice grew progressively; $\mathrm{CpG}$ or mRNA vaccine alone caused mild regression of tumors. To our predicted, the combination of $\mathrm{CpG} 2018 \mathrm{~B}$ and LNP-mRNA resulted in complete regression in melanoma models.

Then, primary tumor cells were isolated and CD8 positive T cells were calculated by FACS. Consistent with the tumor regression, there were more CD8 positive $T$ cells infiltrating in tumor samples (Fig. 4D). HE staining indicated pathology improvement after mRNA vaccine, $\mathrm{CpG}$ and combination treatment(Fig 4E). Moreover, after injection of $\mathrm{CpG}$ and mRNA vaccine, there were up-regulation of CD4 and CD8 positive $T$ cells (Fig $4 \mathrm{E}$ ), suggesting $\mathrm{CpG}$ treatment could promote tumor-infiltrating and effector $\mathrm{T}$ cell populations.

\section{Safety of combined therapy with GpG and mRNA vaccine}

To evaluate the safety and tolerability of mRNA and CpG therapy in syngeneic mouse models, clinical observations and body weights were monitored throughout the study; clinical observations were performed at least 3 times per week. No mRNA or $\mathrm{CpG}$ related clinical findings were identified throughout the entirety of the study. Body weight was similar between all groups during all the time point (data not 
shown). After sacrificed, liver, heart, spleen, lung, kidney were weighted and no significant toxicity phenomena was observed.

Further, clinical chemistry was evaluated at the end of the study in all mice (Fig. 5A-5L). Across all clinical chemistry parameters, no toxicologically relevant findings were detected except a little elevated creatinine level. Importantly, no increase in markers of liver toxicity(ALT, AST) was observed in LNP-mRNA treated mice since the Dlin-MC3-DMA was targeting liver. In consistent, histopathology examination of treatment with $\mathrm{CpG}$ and/or mRNA was performed at the end of the study to further evaluate the safety. No gross pathological findings related to $\mathrm{mRNA}$ or $\mathrm{CpG}$ were observed in liver(Fig. 5M). This result in mice treated with efficacious dose levels of mRNA vaccine and/or CpG is suggestive of a beneficial effect on liver pathology.

\section{Discussion}

Several studies have demonstrated that synthetic CpG ODNs are superb vaccine adjuvants both in mice and human. $\mathrm{CpG}$ ODNs can be divided into three classes, including A-class containing palindromic CpG phosphodiester sequences, which induces very strong IFN-a production but poorly stimulates B cells. In contrast, B-class ODN induces extremely high amounts of IFN- $\gamma$ and promotes B cell proliferation. The Cclass ODN with a stimulatory $\mathrm{CpG}$ plus a palindromic sequence very efficiently induces effects that were previously described to be mediated either by A- or B-class ODN.

In this study we were interested in the identification of an optimal B class CpG ODN for use as an immune activator both in mice and human. At the very beginning, we performed an in-vitro screening of $\mathrm{CpG}$ for the purpose of IFN-y induction. A lot of chemical modifications were added including phosphorothioate (PS), 2'OME and cholesterol. As a result, we found that CpG2018B with PS backbone promotes IFN- $\gamma$ for the best among mice pDCs and 5 human PBMCs. In contrast, other modifications also stimulated IFN- $\gamma$ secretion but not as much as PS only(data not shown), which was consistent with the previously study. An interesting result was that $\mathrm{CpG} 2018 \mathrm{~B}$ shows better activity to stimulate IFN- $\gamma$ secretion compared with the FDA approved ISS-1018 ODN(Dynavax) both in mice and human. For all of these 5 human PBMC, their response to $\mathrm{CpGs}$ varied widely.

Further, our data support the following key mechanistic events mediating the immune-activator effect of CpG2018B. In mice, CpG2018B induces strong TLR9-dependent induction of IFN- $\gamma$, which was abolished by TLR9 inhibition in pDCs. Numerous reports indicated that TLR9 mediated CpG also activating MAPK pathway and stimulates cytokines expression. Here, we also observed a promotion in MAPK substrates phosphorylation and cytokines expression. Knockdown of TLR9 also reduced the production of cytokines including TNFa and IL-6 (Fig 2) but did not change the MAPK pathway (data not shown). One explanation is $\mathrm{CpG}$ activates MAPK via other pathway such as IFNybut not via TLR9. Thus, CpG2018B regulates IFN$Y$, TNFa and IL-6 induction mainly mediated by TLR9.

Considering there is currently being tested in patients with $\mathrm{CpG}$ in melanoma, we hypothesized that a therapeutic effect with locally injected $\mathrm{CpG}$ could benefit this disease. As a result, intratumoral injection 
of $\mathrm{CpG}$ could suppress tumor growth in syngeneic mouse models alone with enhanced immune activity. These data indicate that CpG could turn "cold" tumors into "hot" tumors by stimulating CD4 and CD8 positive T cells population (Fig 3).

A lot of tumor antigen based mRNA vaccine is currently being tested in melanoma patients as a single agent (mRNA-4157, BNT111, etc). We in-vitro transcribed the mRNA of neo-antigen witch was identified previously (Table 1). Then, mRNA vaccine was encapsulated into LNP as describe in the methods. In our study, injection of $\mathrm{CpG}$ and mRNA into the melanoma model results in reduced tumor growth. The result from our studies demonstrates the potency of the treatment for combing $\mathrm{CpG}$ with neo-antigen mRNA vaccine in human melanoma.

Furthermore, the mRNA vaccine was chemical modified by incorporating N1-Methylpseudo-UTP that may provide the advantage of avoiding toxicities that occur with lipid nanoparticles' administration. Our results demonstrated that no significance of ALT or AST levels was increased since DLin-MC3 was a liver targeting cationic lipid. Also, pathology staining of liver indicated no apoptosis or necrosis was found. While there are increased CREA and decreased bilirubin levels in the combo treatment group which need to be further analyzed.

In summary, our results identified a new TLR9 dependent B-class CpG that promotes primary immune response both in mice and human. Further, a combination of $\mathrm{CpG}$ and mRNA cancer vaccine prevents melanoma growth, suggesting a new approach for cancer treatment.

\section{Declarations}

\section{Ethics approval and consent to participate}

Study approval was obtained from the independent ethics committee at the Second Affiliated Hospital of Soochow University (Suzhou, China). The privacy of the patients involved was protected.

\section{Consent for publication}

Study participants provided consent for the publication of the data and any associated images.

\section{Availability of data}

The data that support the findings of this study are available from the corresponding author upon reasonable request. Please contact corresponding author if you want to request the dataset.

\section{Funding}

This study was supported by Suzhou Technology development program (ZXL2018171) and Jiangsu Science and Technology project (BZ2018066) to Rongkuan Hu. The National Natural Science Foundation of China (81873730) and Jiangsu Women and Children Health Key Discipline Program (FXK201758) to Guoqin Jiang, 
Authors' contributions

All authors contributed to data analysis, drafting and revising the article, gave final approval of the version to be published, and agree to be accountable for all aspects of the work

Acknowledgments

We'd like to thank the R\&D team from GenePharma.

Conflict of interest statement

The authors have no conflict of interest to declare.

\section{References}

1. Babu S, Blauvelt CP, Kumaraswami V, Nutman TB: Cutting edge: diminished T cell TLR expression and function modulates the immune response in human filarial infection. $J$ Immunol 2006, 176:3885-3889.

2. Alvarez Y, Valera I, Municio C, Hugo E, Padron F, Blanco L, Rodriguez M, Fernandez N, Crespo MS: Eicosanoids in the innate immune response: TLR and non-TLR routes. Mediators Inflamm 2010, 2010.

3. Suh HS, Zhao ML, Choi N, Belbin TJ, Brosnan CF, Lee SC: TLR3 and TLR4 are innate antiviral immune receptors in human microglia: role of IRF3 in modulating antiviral and inflammatory response in the CNS. Virology 2009, 392:246-259.

4. Lai CY, Liu YL, Yu GY, Maa MC, Leu TH, Xu C, Luo Y, Xiang R, Chuang TH: TLR7/8 agonists activate a mild immune response in rabbits through TLR8 but not TLR7. Vaccine 2014, 32:5593-5599.

5. Byadgi O, Puteri D, Lee JW, Chang TC, Lee YH, Chu CY, Cheng TC: The effect of TLR9 agonist CpG oligodeoxynucleotides on the intestinal immune response of cobia (Rachycentron canadum). $J$ Immunol Res 2014, 2014:273284.

6. Williamson RD, McCarthy FP, Kenny LC, McCarthy CM: Activation of a TLR9 mediated innate immune response in preeclampsia. Sci Rep 2019, 9:5920.

7. Hyer RN, Janssen RS: Immunogenicity and safety of a 2-dose hepatitis B vaccine, HBsAg/CpG 1018, in persons with diabetes mellitus aged 60-70years. Vaccine 2019, 37:5854-5861.

8. Martinson JA, Tenorio AR, Montoya CJ, Al-Harthi L, Gichinga CN, Krieg AM, Baum LL, Landay AL: Impact of class A, B and C CpG-oligodeoxynucleotides on in vitro activation of innate immune cells in human immunodeficiency virus-1 infected individuals. Immunology 2007, 120:526-535.

9. Yamamoto Y, Sugimura R, Watanabe T, Shigemori S, Okajima T, Nigar S, Namai F, Sato T, Ogita T, Shimosato T: Class A Cpg Oligonucleotide Priming Rescues Mice from Septic Shock via Activation of Platelet-Activating Factor Acetylhydrolase. Front Immuno/ 2017, 8:1049. 
10. Wang S, Campos J, Gallotta M, Gong M, Crain C, Naik E, Coffman RL, Guiducci C: Intratumoral injection of a CpG oligonucleotide reverts resistance to PD-1 blockade by expanding multifunctional CD8+ T cells. Proc Natl Acad Sci U S A 2016, 113:E7240-E7249.

11. Sagiv-Barfi I, Czerwinski DK, Levy S, Alam IS, Mayer AT, Gambhir SS, Levy R: Eradication of spontaneous malignancy by local immunotherapy. Sci Transl Med 2018, 10.

12. Sahin U, Kariko K, Tureci O: mRNA-based therapeutics-developing a new class of drugs. Nat Rev Drug Discov 2014, 13:759-780.

13. Malone RW, Felgner PL, Verma IM: Cationic liposome-mediated RNA transfection. Proc Natl Acad Sci U S A 1989, 86:6077-6081.

14. Kreiter S, Vormehr M, van de Roemer N, Diken M, Lower M, Diekmann J, Boegel S, Schrors B, Vascotto F, Castle JC, et al: Mutant MHC class II epitopes drive therapeutic immune responses to cancer. Nature 2015, 520:692-696.

15. Sahin U, Derhovanessian E, Miller M, Kloke BP, Simon P, Lower M, Bukur V, Tadmor AD, Luxemburger $\mathrm{U}$, Schrors B, et al: Personalized RNA mutanome vaccines mobilize poly-specific therapeutic immunity against cancer. Nature 2017, 547:222-226.

16. Huang $H$, Wang $Y$, Li Q, Fei $X, M a H, H u R$ : miR-140-3p functions as a tumor suppressor in squamous cell lung cancer by regulating BRD9. Cancer Lett 2019, 446:81-89.

17. Richner JM, Himansu S, Dowd KA, Butler SL, Salazar V, Fox JM, Julander JG, Tang WW, Shresta S, Pierson TC, et al: Modified mRNA Vaccines Protect against Zika Virus Infection. Cel/ 2017, 169:176.

18. Kulkarni JA, Cullis PR, van der Meel R: Lipid Nanoparticles Enabling Gene Therapies: From Concepts to Clinical Utility. Nucleic Acid Ther 2018, 28:146-157.

19. Pardi N, Hogan MJ, Porter FW, Weissman D: mRNA vaccines - a new era in vaccinology. Nat Rev Drug Discov 2018, 17:261-279.

20. Richner JM, Himansu S, Dowd KA, Butler SL, Salazar V, Fox JM, Julander JG, Tang WW, Shresta S, Pierson TC, et al: Modified mRNA Vaccines Protect against Zika Virus Infection. Cell 2017, 168:11141125 e1110.

\section{Tables}

Table 1. Sequence of CpG oligos, full-strands are phosphorothioate (PS) modified. 


\begin{tabular}{|ll|}
\hline Control (10400DN) & TGACTGTGAACCTTAGAGATGA \\
\hline Cpg2018A & TCGTCGTTTGTCGTTTGACGTT \\
\hline CpG2018B & TCGTCGTTTGACGTTTCGTTTG \\
\hline CpG2018C & TGCTGCTTTGAGCTTTGCTTTG \\
\hline CpG2018D & TGCTCGTTTGAGCTTTCGTTTG \\
\hline CpG1018 & TGACTGTGAACGTTCGAGATGA \\
\hline CpG7909 & TCGTCGTTTTGTCGTTTTGTCGTT \\
\hline SD-101 & TCGAACGTTCGAACGTTCGAACGTTCGAAT \\
\hline
\end{tabular}

Table 2. Prime sequences of qPCR.

\begin{tabular}{|lll|}
\hline Gene & Forward primer & Reverse primer \\
IFN-y & AGGTGCTGCTGATGGGAGG & CGAGTTATTTGTCATTCGGGTGT \\
TNF-a & TCAGCGAGGACAGCAAGG & AGTGAGTGAAAGGGACAGAACC \\
IL-6 & CCTTCTTGGGACTGATGCTG & TTGGGAGTGGTATCCTCTGTGA \\
GAPDH & CCTTCATTGACCTCAACTACATGG & CTCGCTCCTGGAAGATGGTG \\
\hline
\end{tabular}

\section{Figures}


A

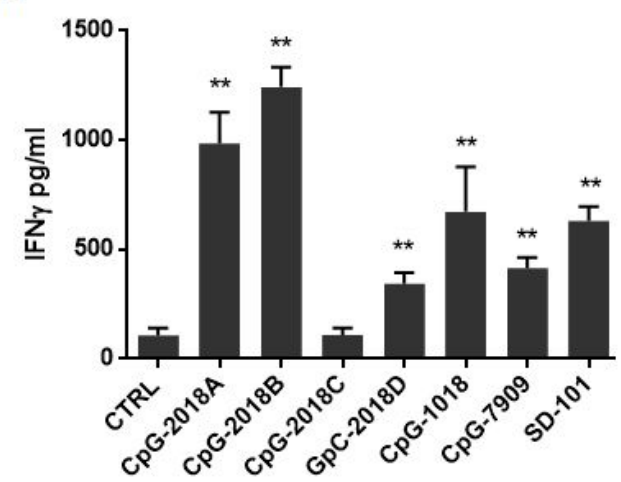

D

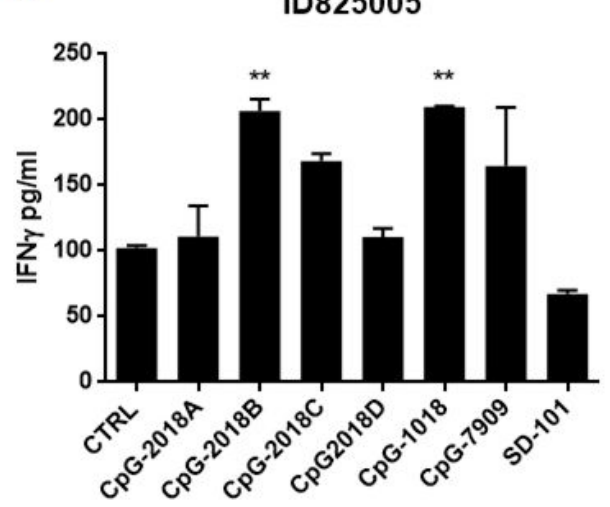

B

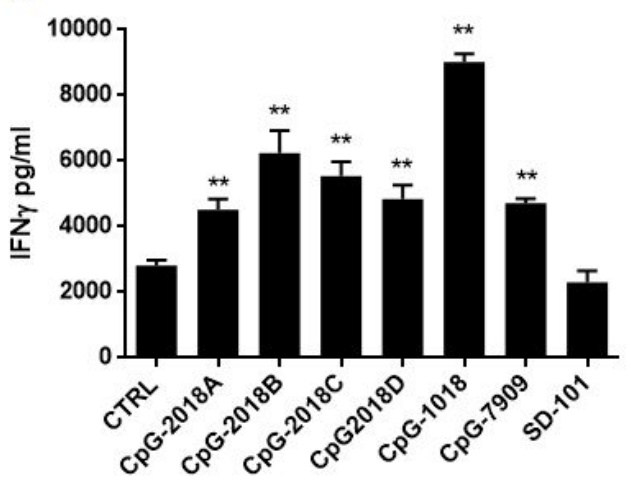

E

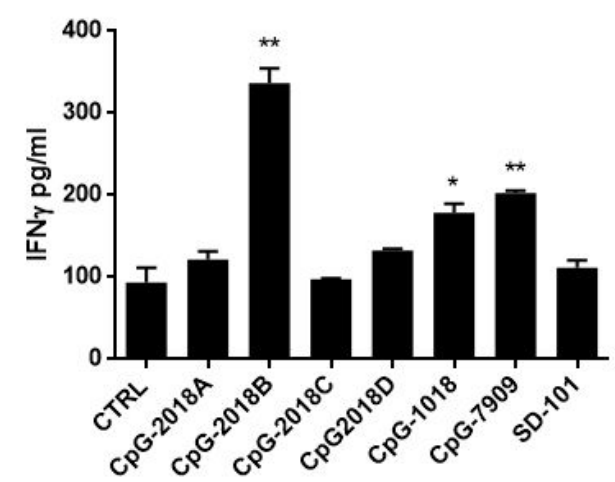

C

ID825130

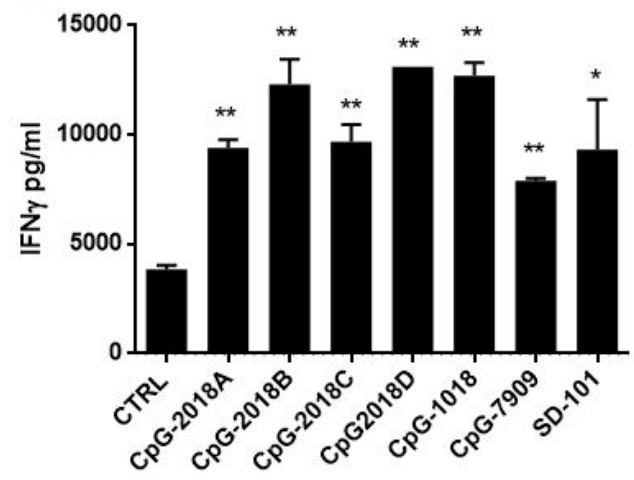

F

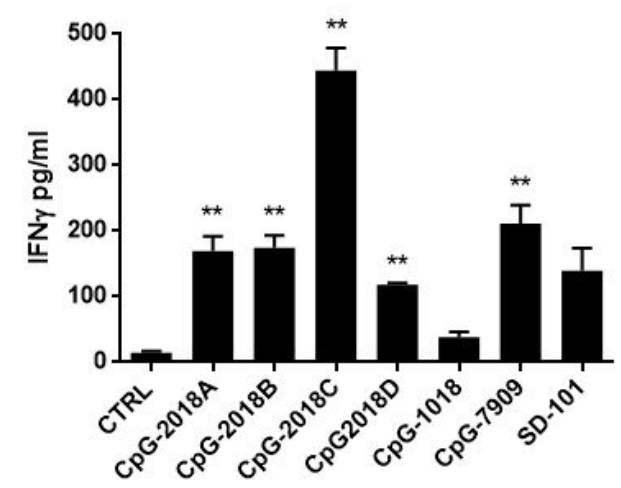

\section{Figure 1}

Identification of CpG2018B promotes IFN-y secretion (A) Identification of CpGs promote IFN-y secretion in mouse pDCs. (B) to (F) CpGs stimulate IFN-y secretion in 5 healthy (non-metastasis tumor) human PBMCs. All the data was compared to the negative control group by student t-test, ${ }^{*} p<0.5 ;{ }^{*} p<0.01$.
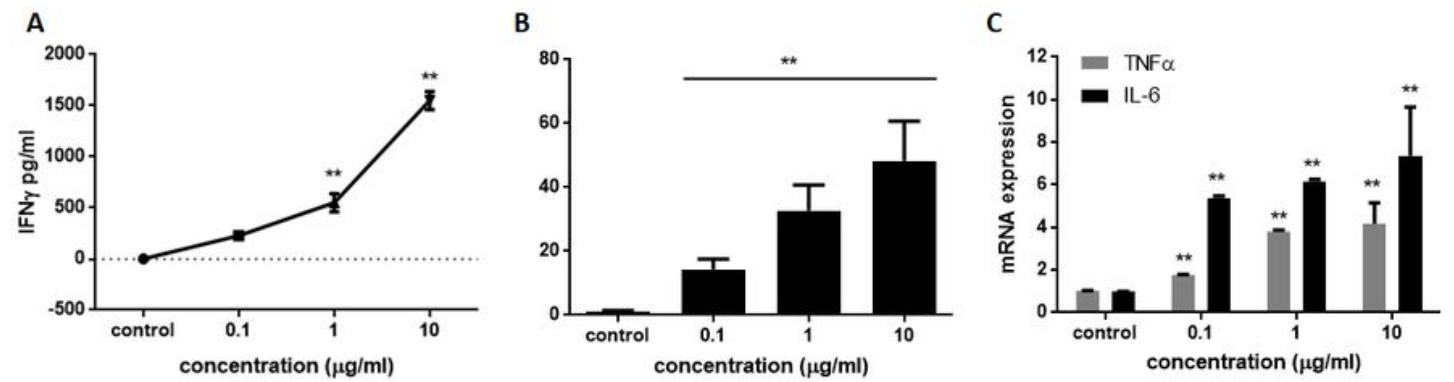

D
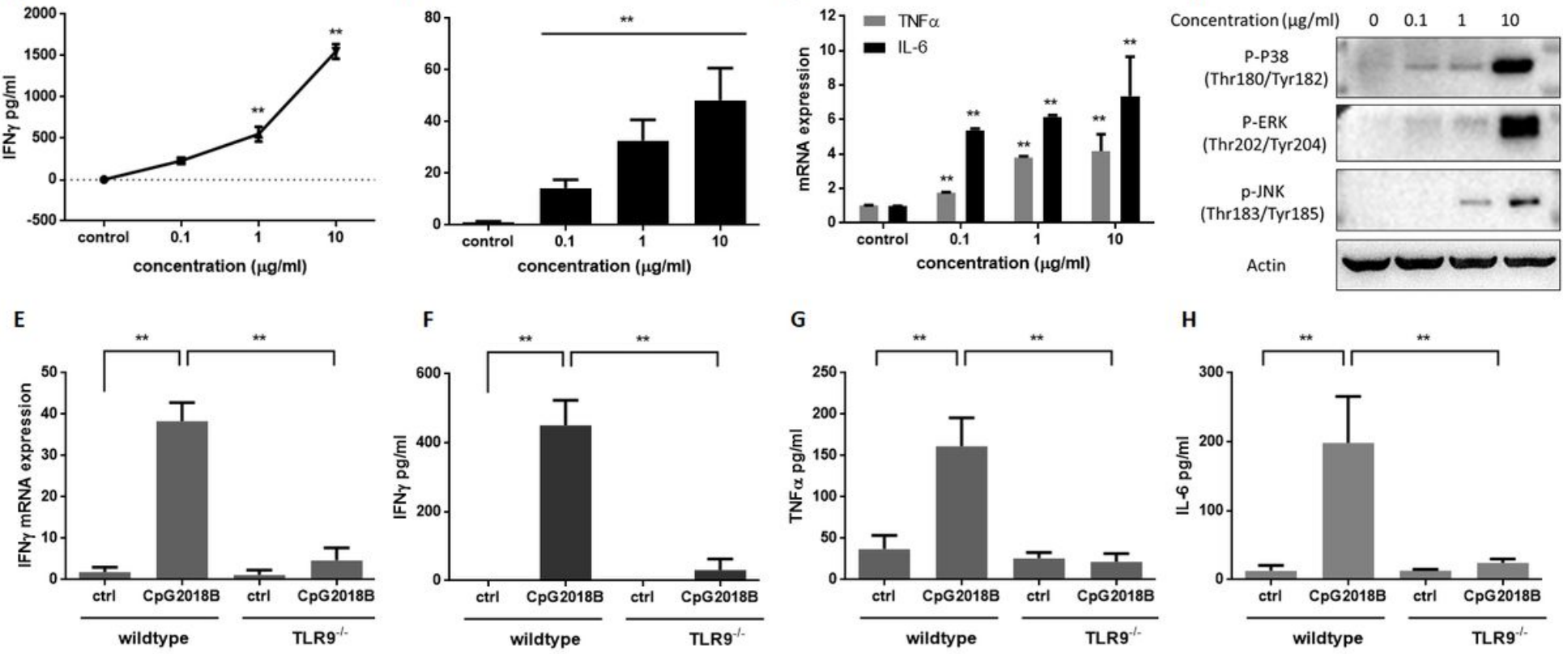

Figure 2 
CpG2018B stimulates IFN- $y$ and cytokines secretion via TLR9 After incubated with CpG2018B for 48 hours, (A) IFN-ywas detected with different concentration of CpG2018B. (B) and (C) IFN-y, TNFa and IL-6 mRNA levels were detected, separately. (D) Phosphorylation of P38, ERK and JNK were detected by western blot. (E) and (F) In TLR9 knockout mice, pDCs were isolated and incubated with CpG2018B for 48 hours. IFN-ysecretion and mRNA levels were detected separately. $(G)$ and $(H)$ TNFa and IL-6 in pDCs were calculated by ELISA. ${ }^{*} p<0.5 ; * * ~ p<0.01$.
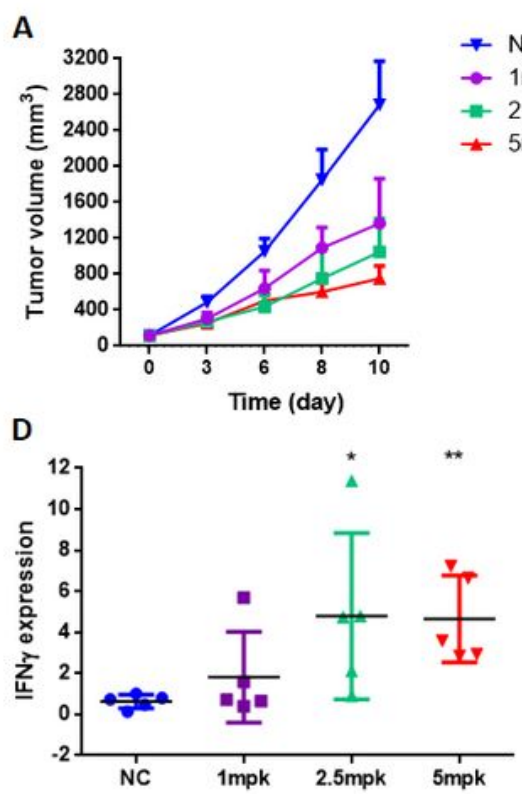

B

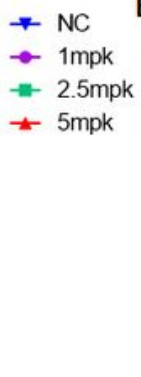

$E$

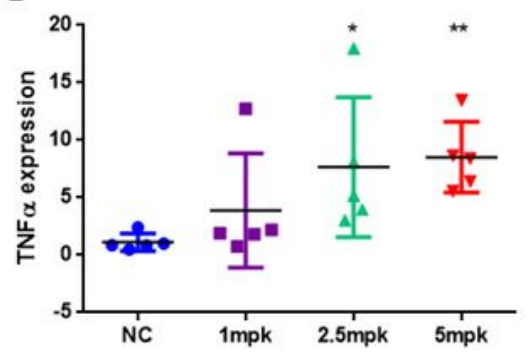

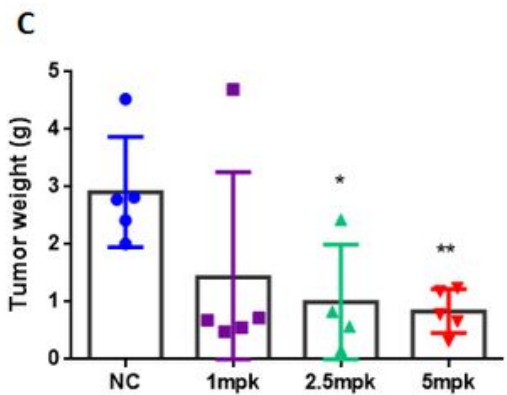

$\mathbf{F}$

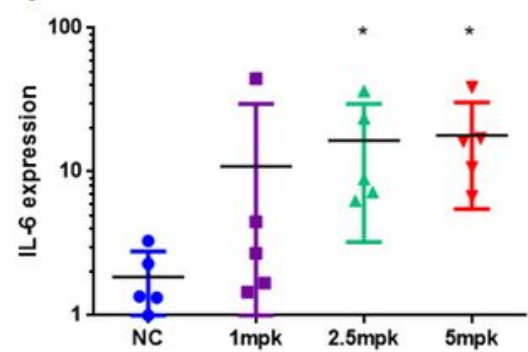

G

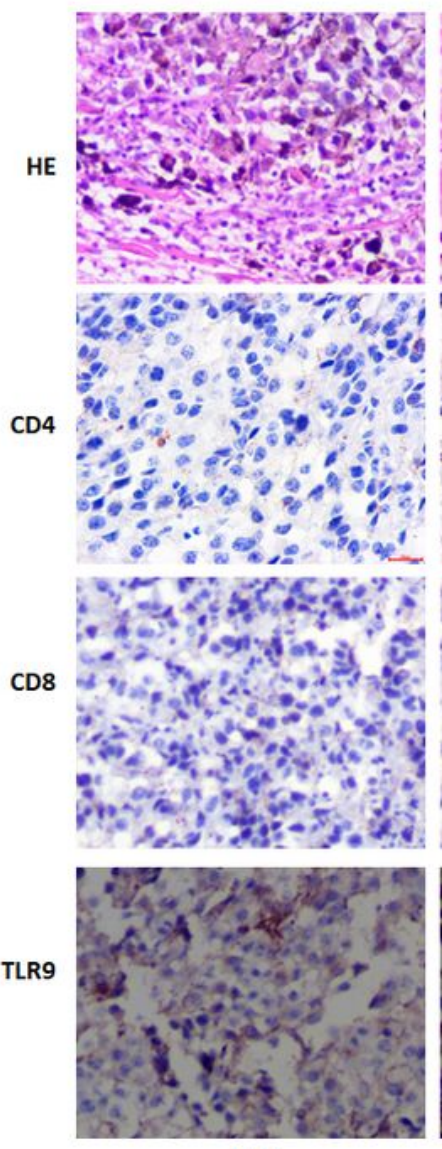

NC
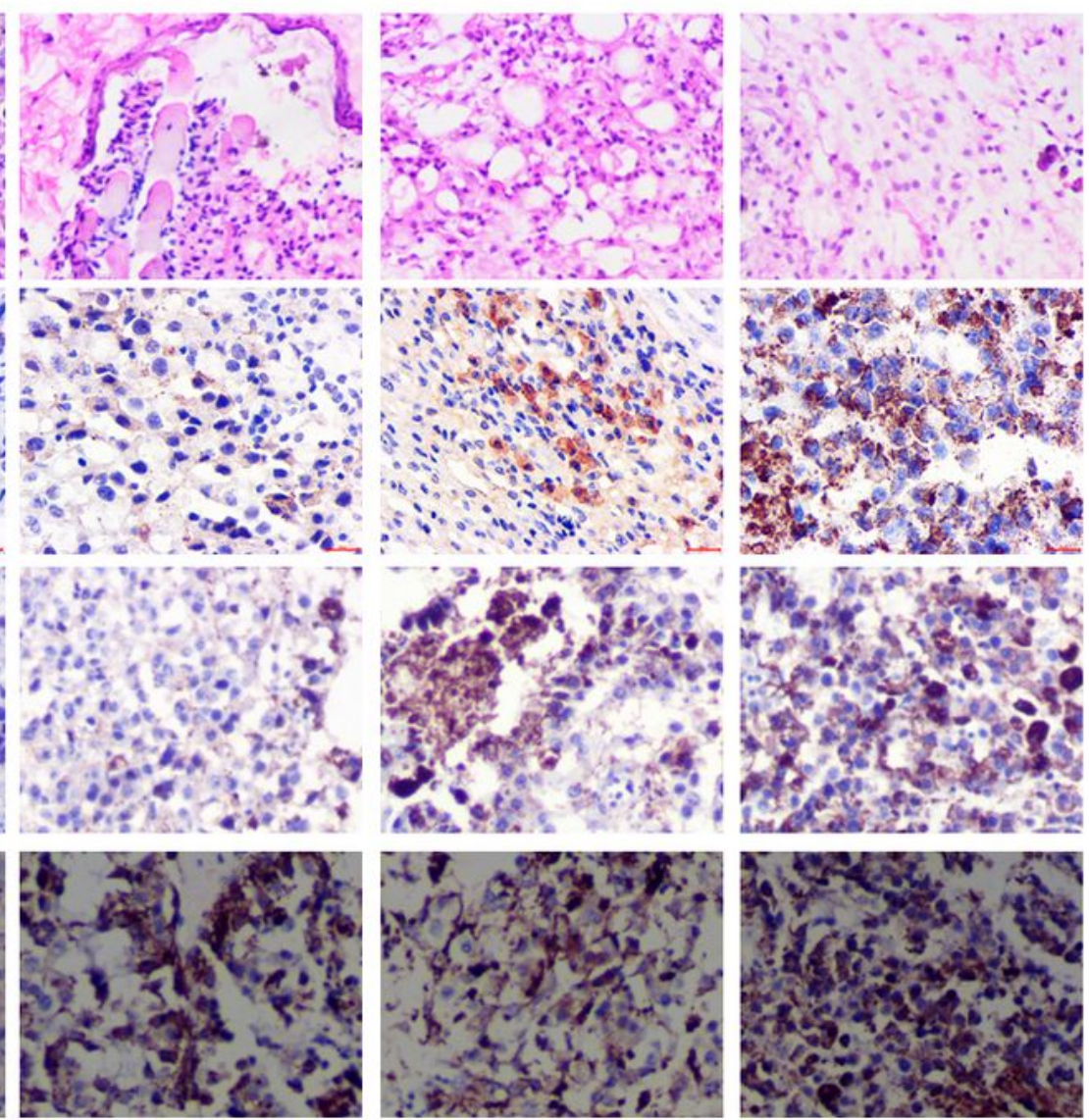

$2.5 \mathrm{mpk}$
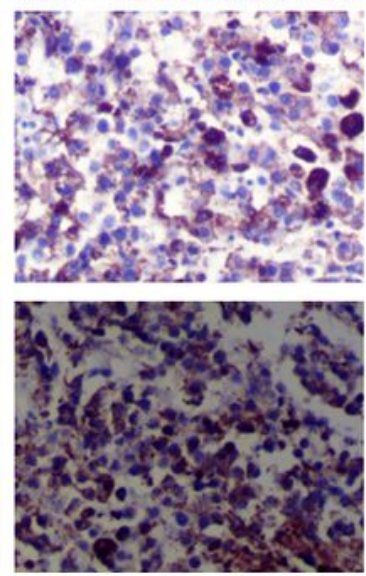

$5 \mathrm{mpk}$ 
CpG2018B induces cytokine expression and tumor regression in melanoma mice models (A) Tumor growth was monitored after intra-tumoral injection of $\mathrm{CpGs}$ in melanoma mice models. (B) and (C) After sacrificed of the mice, tumors were photographed and weighted. (D-F) IFNy, TFFa and IL-6 mRNA levels in tumor samples were analyzed by qPCR. (G) tumors were paraffin embedded and staining with H\&E, CD4, CD8 and TLR9. ${ }^{*} p<0.5 ; * * p<0.01$.

A

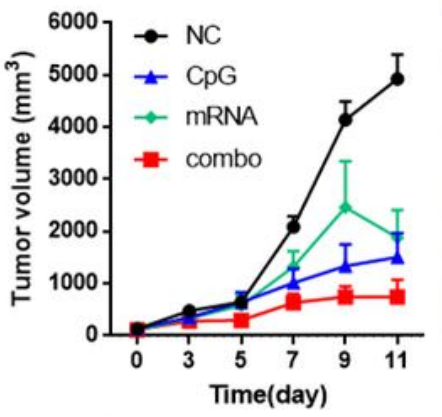

D

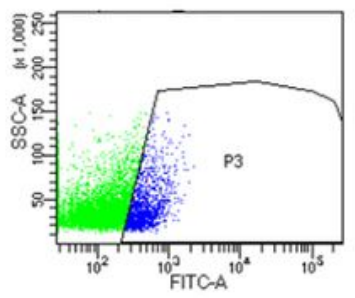

NC

E

HE
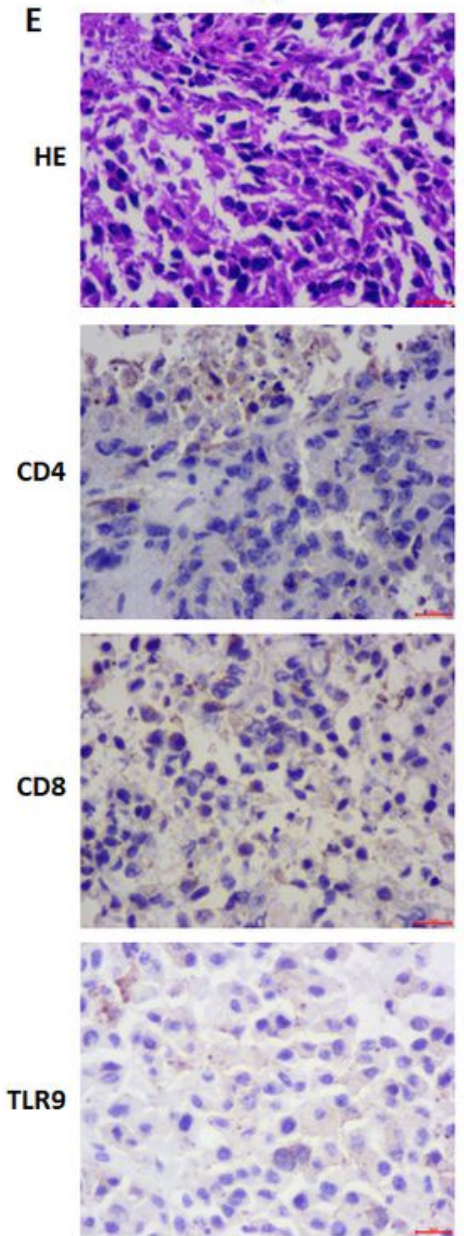

B
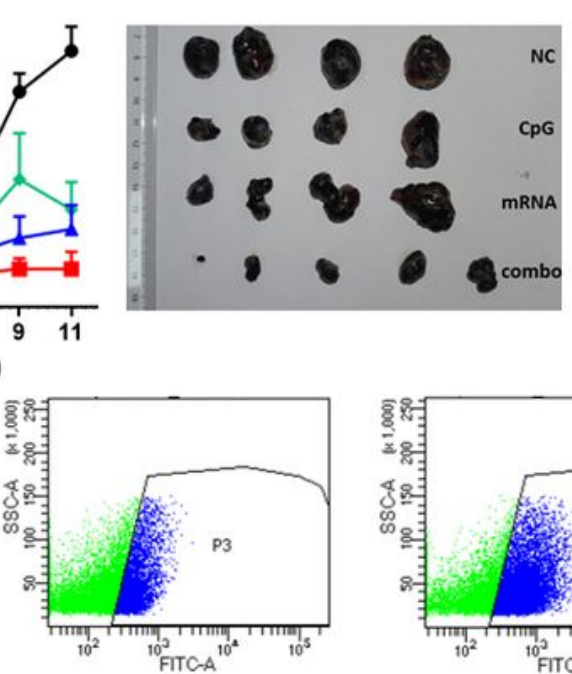

mRNA
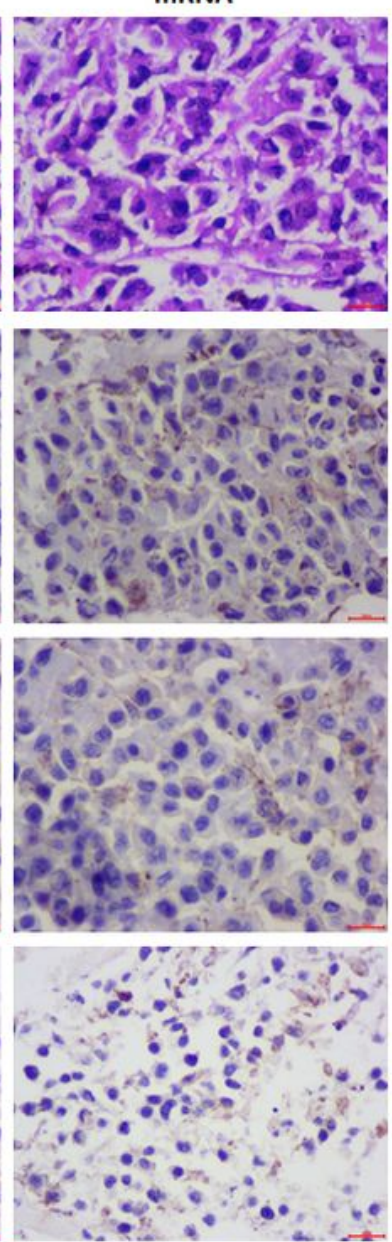

C
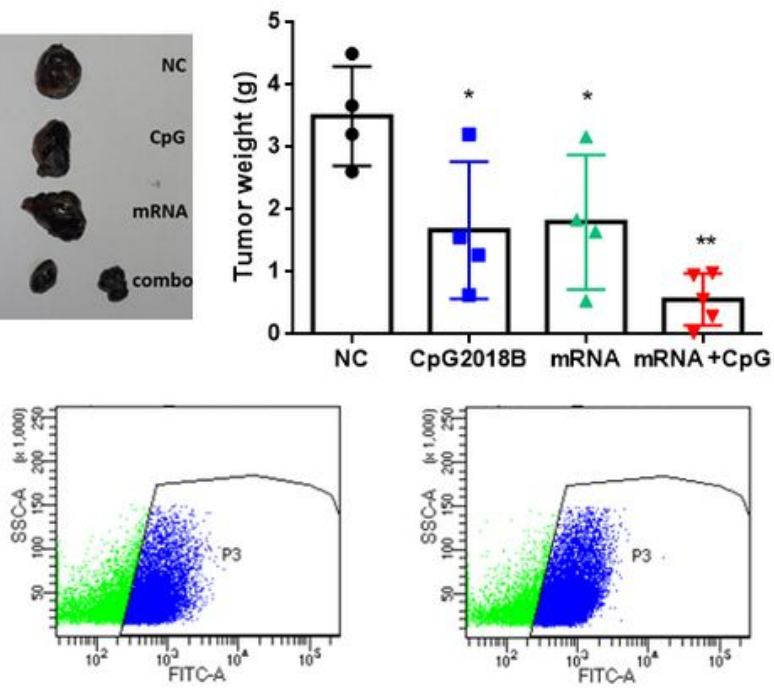

CpG2018B

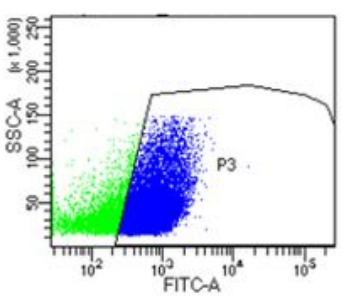

mRNA+CpG
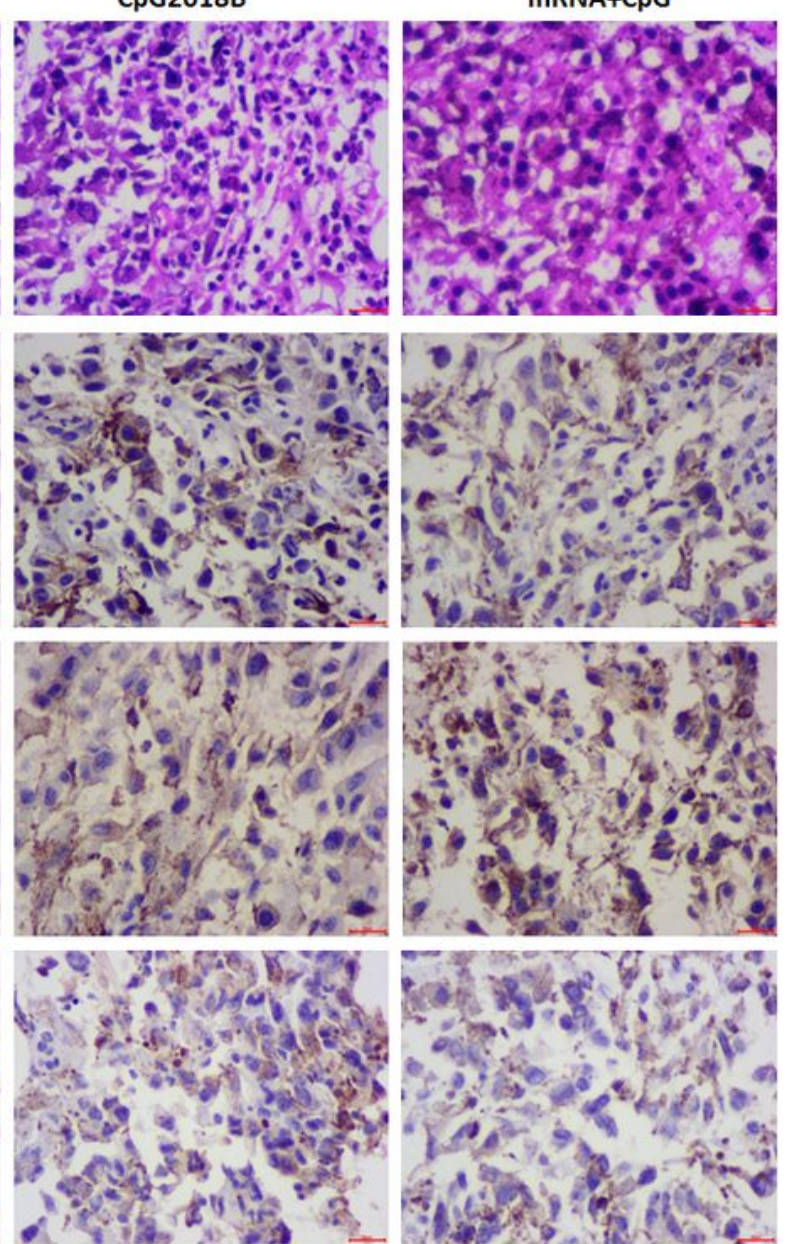

Figure 4 
CpG2018B combine with LNP formulated mRNA vaccine in melanoma models (A) Tumor growth was monitored after intra-tumoral injection of $\mathrm{CpGs}$, mRNA or combo treatment in melanoma mice models $(n=5)$. (B) and (C) After sacrificed of the mice, tumors were photographed and weighted. (D) Half of tumor samples were isolated and CD8 positive T cells were gated by FACS. (E) tumors were paraffin embedded and staining with H\&E, CD4, CD8 and TLR9. ${ }^{*} p<0.5 ;{ }^{* *} p<0.01$.
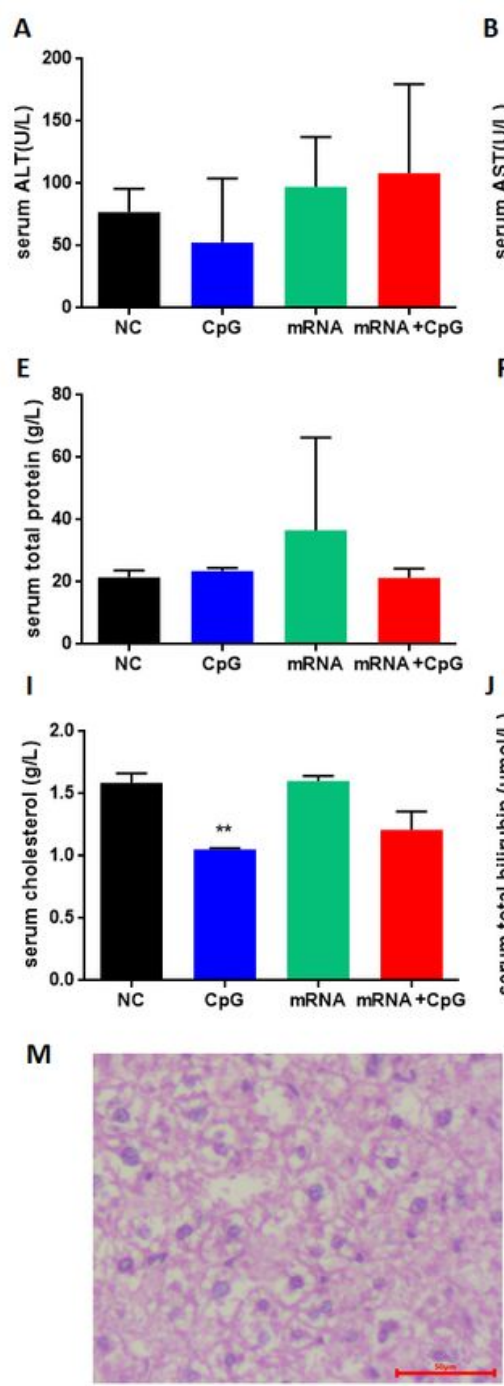

NC
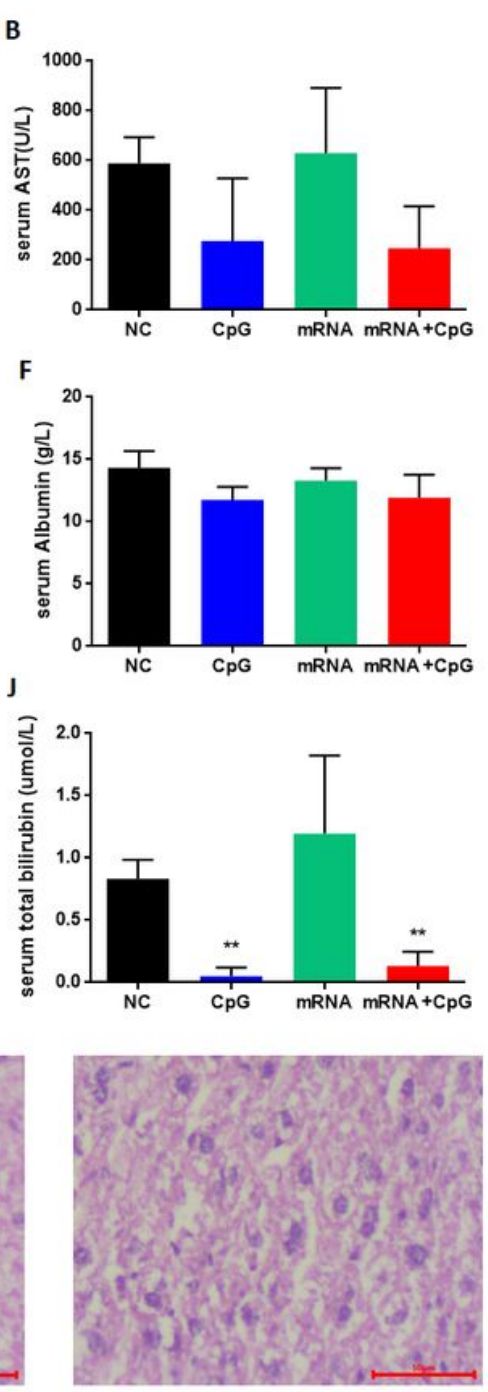

CpG
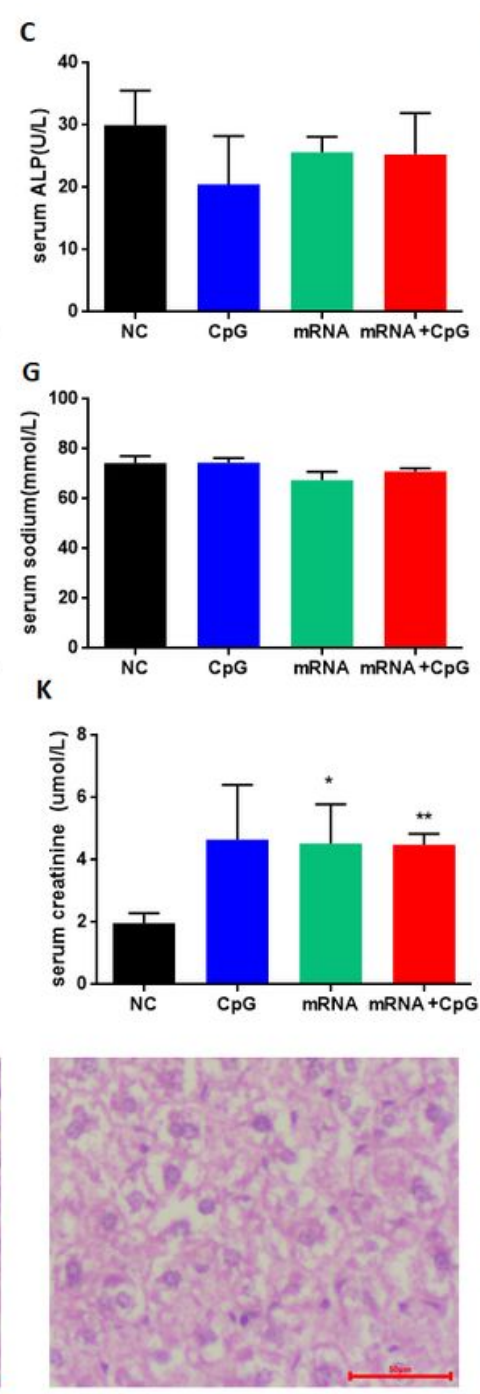

mRNA
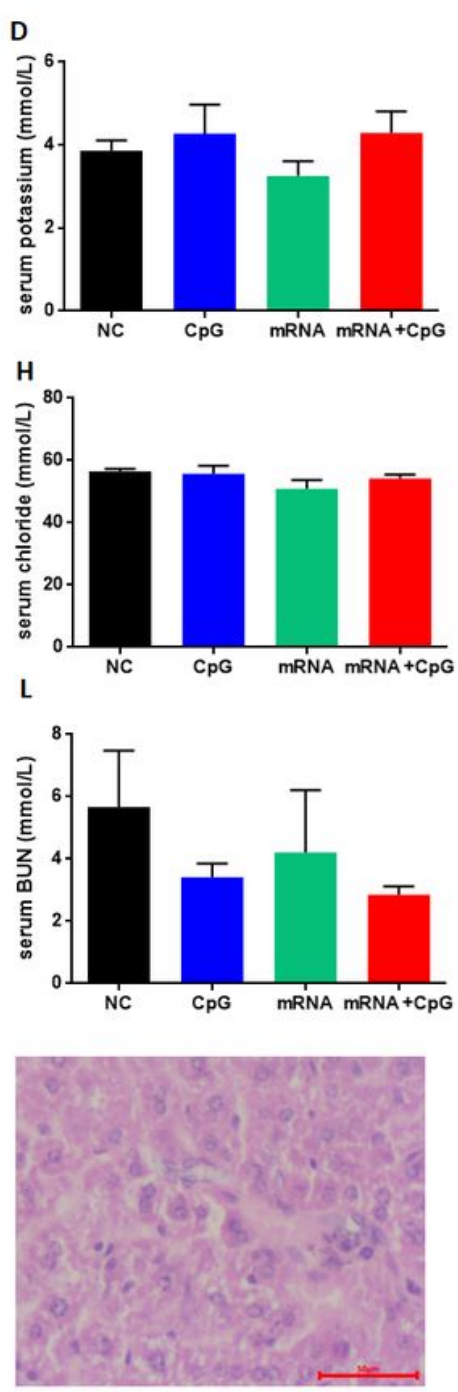

mRNA + CpG

Figure 5

Safety of combined therapy with GpG and mRNA vaccine (A-L) Selected clinical chemistry parameters (serum AST, ALT, ALP, total protein, cholesterol, bilirubin, albumin, etc ) in NC, GpG, mRNA and combo treated mice $(n \geq 4)$. (M) Histology of the liver was examined and representative images of mice injected with $\mathrm{CpG}, \mathrm{mRNA}$ are presented. ${ }^{*} \mathrm{p}<0.5 ;{ }^{* \star} \mathrm{p}<0.01$. 